\title{
Atresia Coanal Bilateral em Paciente de 34 Anos
}

\section{Bilateral Choanal Atresia in 34 Year-old Patients}

\author{
Paulo Tinoco*, José Carlos Oliveira Pereira**, Rodolfo Caldas Lourenço Filbo***, \\ Thiego Silveira Cajubá Brito $* * * *$ Betina Mameri Pereira $* * * *$, Vânia Lúcia Carrara ${ }^{* * * *,}$ \\ Sueliana Marta F. Godoy $* * * * *$.
}

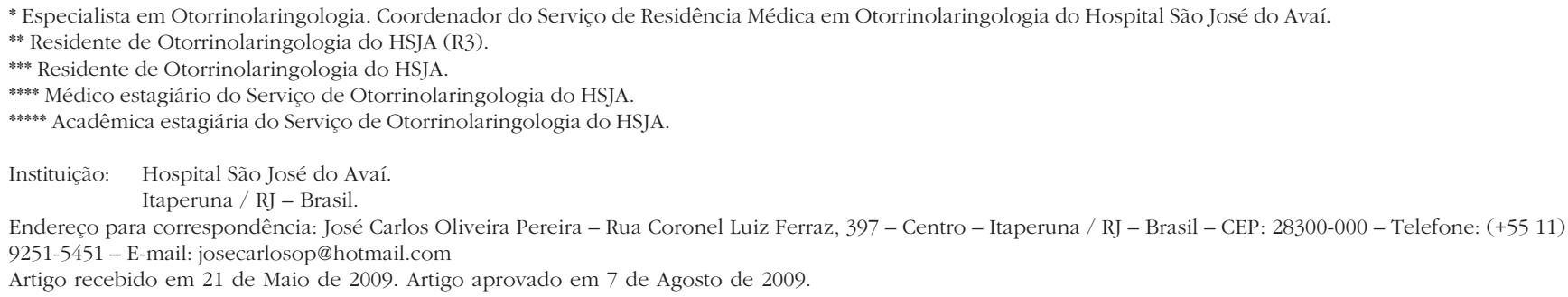

\section{RESUMO}

Introdução:

Objetivo:

Relato do Caso:

Comentários Finais:

Palavras-chave:

\section{SUMMARY}

Introduction:

Objective: Case Report:

Conclusion: Keywords:
A atresia de coanas (AC) pode ser definida como a falha no desenvolvimento da comunicação entre a cavidade nasal e a nasofaringe. Pode ocorrer como uma placa ósteo-membranosa, óssea ou membranosa. O diagnóstico depende de alto grau de suspeição clínica e exames complementares, o tratamento é cirúrgico.

Relatar um caso de uma paciente adulta com diagnóstico de atresia coanal bilateral e que foi submetida a tratamento cirúrgico.

MLSM, 34 anos,sexo feminino, com história de obstrução nasal bilateral, rinorreia hialina desde a infância. Na endoscopia nasal e Tomografia Computadorizada de seios da face evidenciou atresia ósteo-membranosa bilateral. Paciente foi submetida a tratamento cirúrgico endoscópico via transnasal, com confecção de retalho mucoso cobrindo a área cruenta, sem colocação de stent. Foi feito Tomografia de controle que evidenciou coanas abertas e amplas. Paciente evoluiu bem e apresenta respiração nasal normal.

A AC bilateral, embora rara, pode ocorrer em pacientes adultos.

atresia das coanas, obstrução nasal, endoscopia, diagnóstico precoce.

Choanal atresia (CA) can be defined as a failure to develop the communication between the nasal cavity and nasopharynx. May occur as a plate osteo-membranous, bony or membranous. Diagnosis depends on high clinical suspicion and laboratory tests, treatment is surgical.

To report a case of an adult patient diagnosed with bilateral choanal atresia and underwent surgery. MLSM, 34 years old female with a history of bilateral nasal obstruction, rhinorrhea hyaline since childhood. On nasal endoscopy and CT scan of the sinuses showed bilateral membranous atresia osteo. Patient underwent endoscopic transnasal route, with production of mucosal flap covering the wound, without stent placement. Tomography was performed which showed control choanae open and broad. The patient progressed well and has normal nasal breathing.

AC bilateral, although rare, can occur in adult patients.

choanal atresia, nasal obstruction, endoscopy, early diagnosis. 


\section{INTRODUÇÃO}

A atresia de coanas (AC) pode ser definida como a falha no desenvolvimento da comunicação entre a cavidade nasal e a nasofaringe (1). Sua incidência é de 1:5.0008.000 nascimentos, sendo o sexo feminino mais acometido que o masculino, numa proporção de 2:1, os defeitos unilaterais são mais comuns que os bilaterais $(2,3)$. Pode ocorrer como uma placa ósteo-membranosa (70\%), óssea (20\%) ou membranosa (10\%). O diagnóstico depende de alto grau de suspeição, endoscopia nasal e tomografia de seios paranasais, o tratamento é cirúrgico.

\section{Relato do Caso}

MLSM, 34 anos, sexo feminino, com história de obstrução nasal bilateral, rinorreia hialina e, por vezes, mucopurulenta desde a infância. Relata episódios frequentes de rinossinusite. Não sabe relatar se teve problemas respiratórios ao nascer. Ao exame físico, não há associação com outras malformações congênitas. Na oroscopia apresenta protusão dentaria e palato ogival. Endoscopia nasal: confirmação da atresia bilateral (Figura 1). Solicitado tomografia computadorizada de seios da face que evidenciou atresia ósteo-membranosa bilateral (Figura 2). Paciente foi submetida a tratamento cirúrgico endoscópico via transnasal, com confecção de retalho mucoso cobrindo a área cruenta, sem colocação de stent (Figura 3), sendo usado endoscópio rígido de zero grau. Evoluiu sem intercorrências no pós-operatório imediato, fez uso de amoxacilina (500mg VO de $8 / 8$ hs por 10 dias) e lavagem nasal com solução salina fisiológica. Teve alta após 24 horas e foram feitas revisões, através da endoscopia nasal, semanalmente. Foi feito tomografia de controle, no $40^{\circ}$ dia de pós operatório, que evidenciou coanas abertas e amplas (Figura 4). Paciente evoluiu bem e apresenta respiração nasal normal.

\section{DISCUSSÃO}

A atresia de coanas pode apresentar-se como malformação isolada ou, em 20\% a 50\% dos casos, fazer parte de um grupo de malformações congênitas, como: coloboma, malformação cardíaca, retardo mental e de crescimento, anomalias genitais e auriculares ou surdez (4). Sendo assim, um exame físico completo deve ser feito pelo pediatra e otorrinolaringologista para identificar a eventualidade da presença dessas anomalias. Algumas teorias têm sido propostas para explicar a origem embriológica dessa entidade clínica. Dentre as mais aceitas, quatro se sobressaem: 1) persistência da membrana bucofaríngea; 2) falha no rompimento fisiológico da mem-

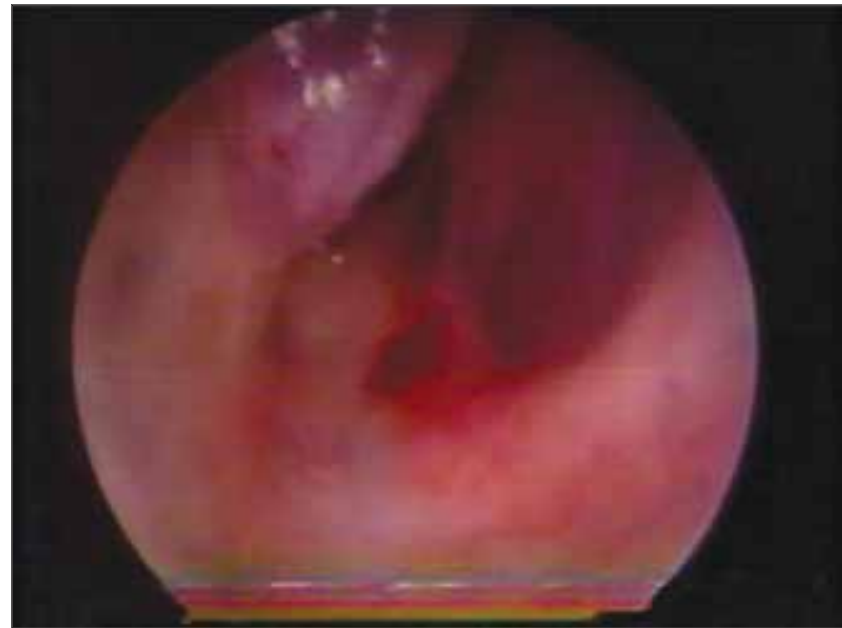

Figura 1. Visão endoscópica de atresia em narina direita.

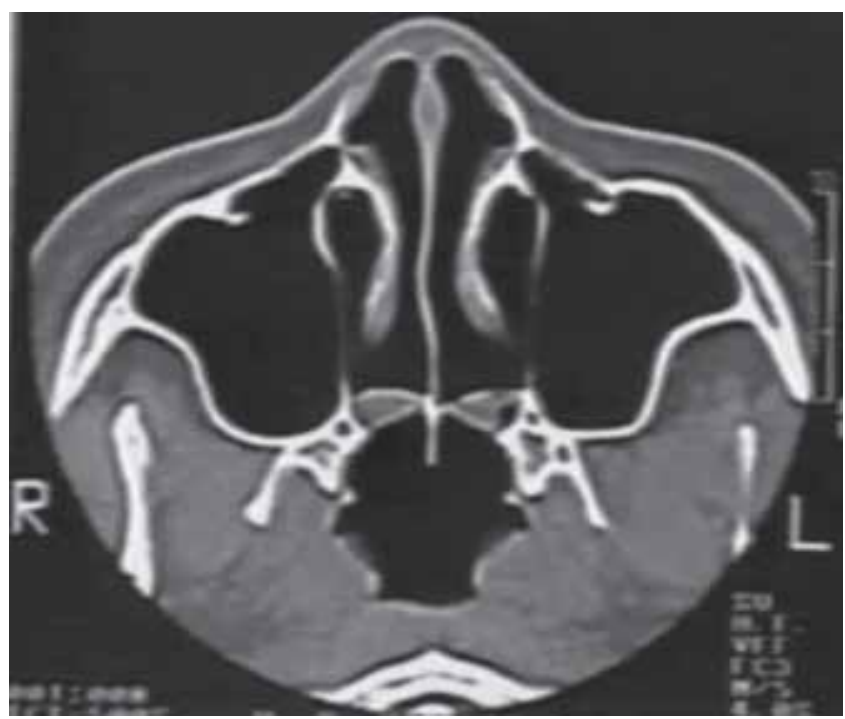

Figura 2. TC de seios da face em corte axial mostrando atresia ósteo-membranosa bilateral.

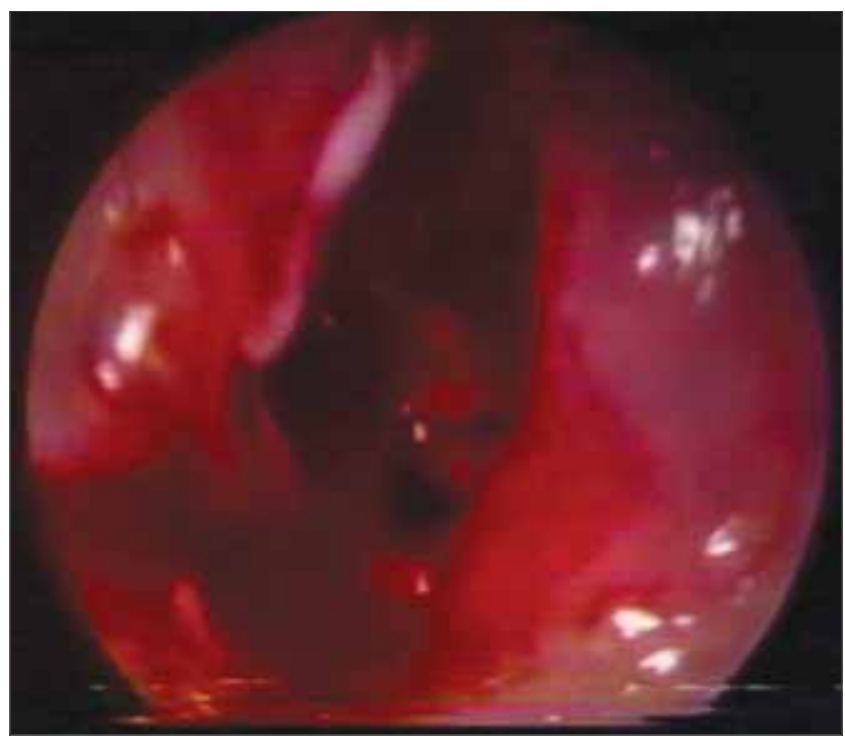

Figura 3. Visão endoscópica de coana direita aberta. 


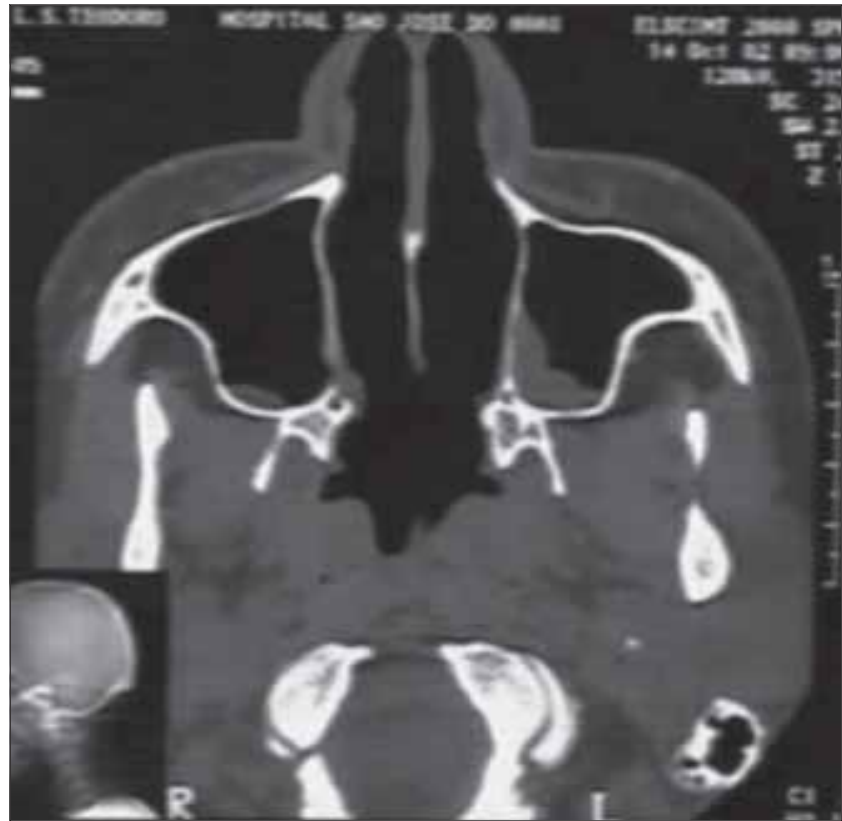

Figura 4. TC de seios da face em corte axial mostrando coanas abertas e amplas

brana buconasal de Hochstetter; 3) aderência anormal de tecido mesodérmico localizado na coana nasal; e 4) crescimento medial dos processos verticais e horizontais do osso palatino $(5,6)$.

O diagnóstico depende de alto grau de suspeição. A AC bilateral cursa com obstrução nasal, estridor, cianose cíclica (melhora com o choro do RN); mas que pode levar à insuficiência respiratória imediata com risco potencial de óbito por asfixia, já que os recém nascidos são respiradores nasais obrigatórios até aproximadamente 3 semanas de vida. Portanto, é uma emergência médica, sendo necessária manutenção de via aérea por intubação oral, traqueostomia ou uso da cânula de McGovern nipple, de modo a estabilizar o paciente e programar o método cirúrgico definitivo. A atresia unilateral pode passar despercebida durante anos e cursar com rinorreia tardia e congestão nasal unilateral; alguns pacientes, contudo podem permanecer oligossintomáticos (7).

Exames complementares como a tomografia de seios da face e a nasofibroscopia são essenciais para a confirmação diagnóstica e planejamento terapêutico avaliação da extensão, localização e distinção das atresias em ósseas, membranosas, e mistas (8). Serve também para fazer diagnóstico diferencial com diversas patologias, como gliomas, hamartomas, cordomas, rabdomiossarcomas, encefaloceles, hipertrofia de vegetações adenoideanas, deformidades septais, dentre outras.

O tratamento inicial consiste na manutenção de via aérea, se necessária, até a correção cirúrgica definitiva, a qual deve restabelecer o fluxo aéreo nasal, evitar danos ao crescimento facial, utilizando uma técnica segura e rápida. Atualmente existem várias técnicas: microscópica e endoscópica via transnasal, transpalatal, transeptal, transantral e dilatações intranasais $(1,7,9,10)$. A técnica transnasal via endoscópica tem sido mais utilizada para correção cirúrgica da atresia coanal, por oferecer boa visualização da área, ser menos traumática, apresentar menores taxas de complicações e proporcionar uma recuperação mais rápida no pós-operatório (11); por isso optamos pela referida técnica no caso relatado.

\section{COMENTÁRIOS FINAIS}

A imperfuração de coana bilateral tem seu diagnóstico realizado mais precocemente devidoà sintomatologia mais exuberante e alto grau de suspeita por parte dos pediatras neonatologistas. É uma doença que coloca em risco a vida da criança, sendo na grande maioria dos casos corrigida nas primeiras semanas. Entretanto, em raros casos, ela pode ser encontrada durante a investigação de adultos com obstrução nasal e rinorreia bilateral, como relatado no caso.

\section{REFERÊNCIAS BIBLIOGRÁFICAS}

1. Lazar RH, Younes RT. Transnasal repair of choanal atresia using telescopes. Arch Otolaryngol Head Neck Surg. 1995, 121(5):517-20.

2. Schwartz ML, Savetsky L. Choanal atresia: clinical features, surgical approach, and long-term follow-up. Laryngoscope. 1986, 96:1335-9.

3. Keller JL, Kacker A. Choanal atresia, charge association, and congenital nasal stenosis. Otolaryngol Clin North Am. 2000, 33(6):1343-51.

4. Tellier AL, Daire VC, Abadie V, AmielJN, Sigaudy S, Bonnet D, Debeney PL, Durand MPM, Hubert P, Michel JL, Jan D, Dollfus H, Baumann C, Labrune P, Lacombe D, Philip N, Lemerrer M, Briard ML, Munnich A, Lyonnet S. Charge Syndrome: Report of 47 Cases and Review. Am Med Genet. 1998, 76(5):402-9.

5. Lorens DC, Casasús JC. Atresia bilateral, ósea, de coanas en adulto. An Otorrinolaringol Ibero Am. 1994, 21(5):487-96.

6. Park AH, Brockenbrough J, Stankiewicz J. Endoscopic versus traditional approaches to choanal atresia. Otolaryngol Clin North Am. 2000, 33(1):77-90.

7. Samadi DS, Shah UK, Handler SD. Choanal atresia: A 
twenty-year review of medical comorbiditied and surgical outcomes. Laryngoscope. 2003, 113:254-8.

8. Schweinfurth JM. Image guidance-assisted repair of bilateral choanal atresia. Laryngoscope. 2002, 112:2096-8.

9. Vogels RL, Chung D, Lessa MM, Lorenzetti FTM, Goto EY, Butugan O. Bilateral congenital choanal atresia in a 13-yearold patient. Int J Pediatr Otorhinolaryngol. 2002, 65:53-7.
10. Josephson GD, Vickery CL, Giles WC, Gross CW. Transnasal endoscopic repair of congenital choanal atresia. Arch Otolaryngol Head Neck Surg. 1998, 124(5):537-40.

11. Cedin AC, Rocha JrFP, Deppermann MB, Manzano PAM, Murao M, Shimuta AS. Transnasal endoscopic surgery of choanal atresia without the use of stents. Laryngoscope. 2002, 112(4):750-52. 